\title{
émulations
}

\section{Lesley J. Wood - Mater la meute. La militarisation de la gestion policière des manifestations}

\section{Hélène Guiery}

Émulations - Revue de sciences sociales

2016, «Comptes rendus critiques, En ligne»

\section{Article disponible à l'adresse suivante}

https://ojs.uclouvain.be/index.php/emulations/article/view/7193

\section{Pour citer cet article}

Hélène Guiery, « Lesley J. Wood — Mater la meute. La militarisation de la gestion policière des manifestations », Émulations, en ligne. Mise en ligne le 24 juin 2016.

DOI : 10.14428/emulations.cr.021

Distribution électronique : Université catholique de Louvain (Belgique) : ojs.uclouvain.be

(C) Cet article est mis à disposition selon les termes de la Licence Creative Commons Attribution, Pas d'Utilisation Commerciale 4.0 International. http://creativecommons.org/licenses/by-nc/4.0/

Éditeur : Émulations - Revue de sciences sociales / Presses universitaires de Louvain https://ojs.uclouvain.be/index.php/emulations

ISSN électronique : 1784-5734

UCL PRESSES

UNIVERSITAIRES 


\title{
Lesley J. Wood - Mater la meute. La mi- litarisation de la gestion policière des manifestations
}

\begin{abstract}
Hélène Guiery ${ }^{1}$
Recensé : Lesley J. Wood, Mater la meute. La militarisation de la gestion policière des manifestations, Montréal, Lux Editeur, 2015, 320 p.

Comme le présentent les premières pages de son ouvrage, Lesley J. Wood est conjointement chercheuse et militante altermondialiste. Diplômée de la London School of Economics et de l'université Columbia, elle est actuellement professeure de sociologie à l'Université York de Toronto. Son ouvrage Mater la meute. La militarisation de la gestion policière des manifestations apporte un nouvel éclairage sur les études du maintien de l'ordre aux États-Unis et au Canada. L'auteure tente d'y retracer les liens entre mondialisation et restructurations néolibérales avec l'évolution de la gestion policière des rassemblements publics. Ainsi, elle identifie « le discours tenu sur l'usage de la force, la chaîne de commandement, les menaces et le rôle de la police dans le cadre d'une analyse des contextes macropolitiques et économiques en mutation » (p. 33) afin de comprendre pourquoi l'utilisation des armes sublétales se renforce en tant que «bonnes pratiques ». Son ouvrage est divisé en huit chapitres allant de la description du monde globalisé à l'étude de cas concret. La richesse de cet ouvrage se distingue dans l'articulation entre ces différentes focales d'analyse. Pour cela, Lesley J. Wood a constitué un corpus varié de reportages, de bulletins des organismes de règlementation, de publications d'agences et organisations policières, de documents de renseignement, de transcriptions de procès ainsi que des notes de terrains ethnographiques. Elle articule finement ce corpus de données aux études antérieures sur la gestion des manifestations.

Après une longue introduction qui retrace l'état de la littérature sur la gestion policière, elle entame son ouvrage par l'analyse des vagues de manifestations de 1995 à 2003. Elle met en perspective les transformations des stratégies de maintien de l'ordre avec celles des mouvements sociaux. Ce premier chapitre retrace l'abandon des stratégies policières de négociations au profit de l'émergence et de la prolifération des unités antiémeutes, des armes sublétales, des arrestations de masse, des contrôles préventifs
\end{abstract}

${ }^{1}$ Doctorante de sciences politiques et sociales, à l'Université Libre de Bruxelles (ULB) et à I'École de Hautes Études en Sciences Sociales de Paris (EHESS). 
et des barrières comme outils pour contrer les manifestations (p. 43). Elle entame cette étude par une énumération des rapports sociaux structurels qui poussent la police à multiplier ses partenariats privés (chapitre 2). S'appuyant sur une comparaison entre quatre grandes villes (Montréal, Toronto, New York et Washington), elle s'intéresse ensuite aux variations locales des stratégies policières dans un contexte mondialisé. L'auteure présente en quoi les conflits locaux configurent l'intégration, l'adaptation et l'expérimentation de nouvelles tactiques de gestion des manifestations en les facilitant ou en les freinant (chapitre 3). Pour présenter ce raisonnement, elle prend exemple sur l'intégration du gaz poivre à l'arsenal policier malgré les controverses qu'il suscite auprès du public (chapitre 4). L'auteure s'appuie ensuite sur l'exemple de la gestion policière du mouvement Occupy — mouvement de protestation contre la domination économique et les inégalités sociales - pour analyser les prises du secteur privé sur la diffusion d'un modèle de gestion des manifestations (chapitre 5). Elle propose dans les chapitres suivants (6 et 7) deux arguments plus détachés de l'évolution néolibérale et mondialisée des sociétés capitalistes. La militarisation de la police est alors envisagée sous le joug de l'amplification et la systématisation de la qualification des manifestants comme «menaces à mater », sous-tendue par les discours politiques de peur et d'insécurité. Elle distingue un basculement des catégorisations, qu'elle conçoit comme des représentations, après les attentats du 11 septembre 2001. Les manifestants rentrent peu à peu sous le même régime d'identification que les terroristes, comme une menace à l'ordre public (chapitre 6). En outre elle explique que cette nouvelle qualification des manifestants est en partie due aux récits colportés par les agents de police dans l'incorporation de la culture policière (chapitre 7). En guise de dernier chapitre, Lesley J. Wood conclut sur des perspectives assez pessimistes concernant la brutalité policière aux vues de l'évolution des sociétés capitalistes.

Sur une dizaine d'années, Lesley J. Wood décrit l'émergence d'un modèle policier qui préconise l'introduction d'armes sublétales, de barrières, d'infiltrations et d'arrestations sélectives et/ou massives. Elle explique que les forces de l'ordre optent pour la stratégie de "neutralisation stratégique » lorsque les organisateurs de manifestations ne sont pas prêts à négocier avec elles. Si la police ne parvient pas à stopper le déroulement d'une manifestation, les manifestants sont généralement perçus comme «menaçants » ou « imprévisibles ». Elle aura alors rapidement recours aux armes sublétales, aux arrestations massives et aux unités antiémeutes. Au fil du temps, les activités militantes seront appréhendées comme des actes criminels auxquels la police fait face par la brutalité envers les manifestants et des décisions abusives quant à l'appréciation de la dangerosité de la situation. Une seconde dimension de la tactique de neutralisation stratégique se déploie parallèlement à partir de 1997. Elle consiste au recours de trois méthodes: 1/ l'érection d'enclos en amont de la manifestation, conçue comme «zone d'expression » de la contestation encadrée par la loi et les forces de l'ordre, 2/ la constitution de périmètres de sécurité autour des lieux de réunions internationales pour les protéger des manifestants perçus comme une menace à leur bon déroulement 
et 3/ l'encerclement de la foule pendant la manifestation, consistant à enfermer la population à l'aide de barrières et les y contenir un certain laps de temps. Ces trois méthodes permettent de légitimer et de légaliser des arrestations qui pourraient être condamnées d'abusives dans d'autres cadres. C'est dans les années 2000 qu'apparaissent les arrestations préventives. Elles répondent à l'accusation de trouble à l'ordre public qui permet de retenir des manifestants, sans preuve avérée et sans motif, pendant 24 heures. La neutralisation stratégique se fonde en dernier lieu sur le mode de contrôle, plus ancien mais toujours d'actualité, des infiltrations menant à des accusations de complot ou d'incitation à l'émeute qui peuvent aller jusqu'à des peines de prison ferme.

Les évolutions en matière de gestion policière, tout comme la majorité des politiques publiques actuelles, vont dans le sens d'une réduction de leurs financements qui implique le développement de justifications et de preuves d'efficience. Pourtant, quels que soient les contextes et les difficultés à gérer les crises sociales, la police doit maintenir sa légitimité, son influence et son financement, car c'est en partie sur ces dimensions que repose le bon fonctionnement de l'État. Pour pouvoir les garantir, les services de police se tournent vers de nouveaux acteurs tels que les réseaux professionnels, le secteur privé ou les autorités civiles. Ainsi, se développent une plus grande importance des partenariats public-privé et l'usage croissant des technologies de communication et d'information dans la gestion de prises de décision. Pour maintenir leur autonomie, leur légitimité et leur capital économique, les corps policiers sont contraints de recourir à quatre mécanismes repérés par l'auteure : 1 / la résistance à l'influence des entités qui les surveillent; 2 / la réduction des sources internes de divisions ; 3 / la formation d'alliances avec des sources externes de légitimité; 4/ l'introduction d'innovations certifiées par des experts policiers. Ces mécanismes varient selon le contexte local et la position occupée par l'agence dans le champ policier plus large. L'une des grandes qualités de l'ouvrage se présente ainsi dans l'articulation des différents secteurs privés et politiques avec l'organisation et le fonctionnement des agences de police. Cependant, elle ne prend que très peu en considération les actions concrètes des policiers.

Bien que Mater la meute s'ouvre sur un récit ethnographique du contre-sommet antiG20 à Toronto, la place de l'expérience de la gestion policière est minime. L'une des visées de l'auteure est pourtant de «donner aux mouvements sociaux la capacité de résister à la domination de l'État et du monde des affaires, dépasser les explications primaires et porter un regard analytique sur les services de police» (p. 19). Sa position extérieure à l'action réelle de la police rend cette ambition difficile à garantir.

Lesley J. Wood indique que les cas de brutalité policière ou d'arrestations abusives lors de manifestations sont souvent l'occasion pour la police d'intégrer et de rendre légitime de nouvelles pratiques et technologies jusqu'alors controversées. Le Taser a pu ainsi être intégré à l'arsenal policier et légitimé au sein du corps policier, car il a été présenté comme alternative à la violence de la matraque. Bien qu'elles soient soutenues plus ou moins explicitement par les pouvoirs politiques ou les experts d'opinion, les 
abus en matière d'utilisation d'armes sublétales et de brutalité tendent à isoler les agences de police qui doivent s'en justifier auprès du grand public. Le manque fréquent de reconnaissance du grand public ou de soutien de la part des sphères politiques conduit les agences de police à ressentir un sentiment d'isolement. Pour y pallier, elles tendent à renforcer leur identité et la diffusion de « bonnes pratiques » afin d'acquérir une meilleure légitimité. L'intégration de nouvelles pratiques comme solution aux problèmes de la gestion policière des manifestations se répand grâce à « l'idée d'une identité professionnelle partagée au sein des agences de police » (p. 184). Le gaz poivre a vu son utilisation se systématiser comme outil privilégié de la gestion des manifestations à partir des années 2000. La persistance de cette pratique, malgré l'indignation publique et les poursuites judiciaires, s'explique pour Lesley J. Wood par deux processus : la certification et l'identification. Elle désigne la certification comme "le mécanisme par lequel des experts respectés proclament l'utilité d'une innovation » et l'identification comme «le processus par lequel les adoptants potentiels d'une innovation se considèrent comme semblables aux utilisateurs précédents » (p. 146). Elle considère que ces deux processus sont facilités grâce au partage d'une identité forte qui se développerait notamment par la diffusion rapide d'anecdotes, souvent déformées, concernant la violence des manifestants.

Lesley J. Wood analyse les récits sur « les fusils à eau remplis d'urine» (p. 219) des manifestants comme la constitution d'un savoir qui s'incorpore à l'habitus de la police grâce à la socialisation - le fait que le récit se raconte de bouche à oreille et crée des affinités entre les agents de même culture et à la formation. Le récit est diffusé pour justifier des stratégies policières ou interpréter les interactions entre agents. Ces récits ont pour conséquence de déshumaniser et de dépolitiser les manifestants en relayant leurs actions comme violentes, préméditées et intentionnelles. L'auteure considère les effets de ces récits comme l'alimentation d'une peur qui justifie l'emploi de l'équipement antiémeute et l'anxiété dont sont victimes les policiers.

Lesley J. Wood choisit le modèle d'analyse de Pierre Bourdieu pour comprendre les dynamiques de légitimation des «bonnes pratiques » en matière de gestion policière des manifestations. Bien qu'elle évoque Boltanski, Thévenot ou encore Beck et Giddens, ces auteurs ne semblent pas servir l'analyse de l'objet d'étude, mais l'illustrer pour expliquer le contexte sociopolitique des sociétés contemporaines gouvernées par l'anticipation du risque. La constance des conclusions de l'analyse de Wood rend la lecture quelque peu redondante tout en laissant le lecteur avec un certain malaise. À force d'expliquer la gestion violente des manifestations par les conséquences de la mondialisation et de la restructuration néolibérale, l'intention policière individuelle n'est jamais prise en considération pour expliquer leurs actions. Les policiers sont présentés comme dominés par un système sur lequel ils n'ont pas de prise, ce qui conduit à penser au fil de la lecture qu'il faudrait presque les excuser d'être violents, pour cause de dysfonctionnement du système social, politique et économique. La brutalité policière et 
les arrestations abusives sont présentées comme des actions survenant de manière mécanique, sous le poids d'une nécessité externe déterminée par la position sociale des policiers au regard de l'État. La brutalité et les arrestations ne sont jamais décrites «comme des stratégies déployées par des individus poursuivant des projets volontaires, conscients et libres» (Ogien, 2007: 32). L'intégration et la légitimation de « bonnes pratiques », squelette à partir duquel s'organise l'analyse présentée, sont ainsi saisies comme des objets objectivés dont la réalisation dépend de l'acceptation des contraintes déterminées en extériorité de leurs usages concrets.

Le recours au concept d'habitus semble aussi problématique pour comprendre l'activité policière de gestion des manifestations. Lesley J. Wood explique que les récits colportés participent à la constitution d'un savoir qui s'incorpore à l'habitus policier, justifiant alors leurs pratiques. Le lecteur peine à comprendre par quels processus observables et descriptibles, un récit, qui susciterait la peur et l'anxiété des policiers, pourrait participer à justifier l'usage d'armes sublétales contre des manifestants non armés. Ces apories semblent liées à la référence permanente au « champ » imaginé par Pierre Bourdieu (1980), qui revendique une position objectiviste. Contrairement à la démarche de Cédric Moreau de Bellaing (2016) qui s’intéresse aux pratiques policières de l'intérieur même de leurs accomplissements, Lesley J. Wood conserve une extériorité analytique tout au long de son étude. Les policiers n'y agissent qu'en fonction des limites prédéterminées par le champ policier dans lequel ils évoluent et sans jamais remettre en cause les mobiles personnels qui guideraient leurs actions. Malgré les grandes qualités que présente l'ouvrage, le lecteur qui s'attendait à ce qu'il donne « aux mouvements sociaux la capacité de résister à la domination de l'État et du monde des affaires» (p.19) reste quelque peu sur sa faim. En introduction, l'auteure se décrit comme manifestante. Elle livre quelques bribes ethnographiques sans pour autant les analyser en tant que telles. Jusqu'à la fin de l'ouvrage, le lecteur attend une analyse de l'intérieur même des pratiques observées et décrites. Ces analyses auraient permis une attention plus fine à l'imbrication des enjeux sociopolitiques de la militarisation de la gestion des manifestations, de l'influence d'un système globalisé et néolibéral aux pratiques concrètes, en train de se faire, des agents de police. Cependant, il est tout fait concevable qu'une analyse de ce type soit méthodologiquement difficilement envisageable.

\section{Bibliographie}

BourdiEu P. (1980), Le sens pratique, Paris, Éd. Minuit («Le sens commun »).

MOREAU DE BELLAING C. (2016), Force publique, Une sociologie de l'institution policière, Paris, Économica («Études sociologiques»)

OGIEN A. (2007), Les règles de la pratique sociologique, Paris, PUF. 